\title{
Psychological Problems among the Health Workers During Pandemic Covid-19
}

\author{
Astabrak A. N. Al-Hamoodi ${ }^{1}$, Burhan Hadi ${ }^{2}$, Kafi Mohammad Nasir Al Asadi ${ }^{3}$ \\ ${ }^{1}$ Asst. Lect., Master in Mental and Psychological Health, University of Kufa-College of Nursin, ${ }^{2}$ Asst. Lect., \\ Master in Mental and Psychological Healthm Altoosi University College, ${ }^{3}$ Prof., Ph.D. Community Health in \\ Nursing Department, Altoosi University College, Iraq
}

\begin{abstract}
The COVID-19 is a globally problem influences in all function of life, from health to economic and political of countries. It is still to elevated in incidence and killed people specially among health workers. The study aimed to assess the psychological problems among health workers who work in health facilities at Babylon Province.

Descriptive-analytic study conducted during the era of COVI-19 by using online GHQ-12 items as instrument to measure the symptoms of psychological problems. Convenience sample included 220 health workers participated in the study. The study revealed elevated in psychological problems among health workers and found significant relationship between gender and psychological problems. The study concluded to the support and available the enough training and medical health equipment may be reduced it.
\end{abstract}

Keyword: Psychological problems, health workers, pandemic Covid-19.

\section{Introduction}

The COVID-19 is a global outbreak is a highly contagious and it is main cause of respiratory illness. ${ }^{[1]}$ It is a viral infection recognized by fever, shortness of breath, cough and chest infection. ${ }^{[2]}$ First detect to it in Wuhan, China ${ }^{[3]}$, and rapidly spread in all world yet ${ }^{[1]}$. But COVID-19 is becoming an increasing public event being a rapid epidemic ${ }^{[4]}$. The World Health Organization states in the official website (March, 2020), there is 100,000 person and more are diagnosed with COVID-19 infection around of world ${ }^{[5]}$. So that any person can be affected emotionally and exposure to psychological problems symptoms during of the COVID-19 now. Most communities and people had overwhelming feelings of fear, stress and anxiety about a COVID-19 disease

\section{Corresponding Author:}

Astabrak A. N. Al-Hamoodi

Asst. Lect., Master Degree in Mental and Psychological Health, University of Kufa-College of Nursing e-mail: astabrka.alhamoody@uokufa.edu.iq
[6], but there is evidence reported to proof the health workers are effected by the psychological problems after the COVID-19 reach to the maximum point such as stress, anxiety, insomnia and other health problems. ${ }^{[7]}$ Everyone responds to stressful circumstances and reacts differently according to experiences, abilities to adapted with it and how coping with emotional distress ${ }^{[8]}$ due to the highly risks of exposure or ethical dilemma and stress of workload and work hours ${ }^{[9,10,11]}$.

Sadly, some healthcare workers can face rejection because of stigma or fear by their families or friends, so it may be make them which is a victim and increased the psychological problems on them ${ }^{[12]}$.

The study goals to assess the symptoms of psychological problems among the health workers during pandemic Covid-19 at Babylon Province and find out the relationship between their characteristics and the symptoms of psychological problems among them .

\section{Material and Method}

The descriptive-analytic study design conducted at A Nonprobability convenience sample was conducted in 
Babylon Province during the era pandemic Covid-19 in world generally and in Iraq especially from 20 March to 5th April 2020 by using Google samples forms online to self-reported questionnaire distributed on 220 participants (57 female and 163 male) from general health workers (24500) were work in all health care facilities in Babylon Province (see table (1)). After get consent to participated in study. The study sample is a non-probability convenience sample, included participants from all governmental health facilities of Babylon Province weather worked in isolated facilities of COVID-19 or in another governmental health facilities.

The study questionnaire had two parts. Part one related to demographic characteristics of health workers like age, gender, experiences duration with contagious diseases, level education, marital status. Part two related to General Health questionnaire (GHQ12-items) to explore the psychological problems in health workers who are not have psychological problems by selfreported. The questionnaire is translated and use in AlHamoody study (2019) ${ }^{[13]}$ and before him it used for first time by Goldberg (1970) was use GHQ to assess psychological well-being. It has four scores (0-3) begin from much less than usual to better than usual. It has cutoff point 1.5 , when a person had $\geq 1.5$ that mean had psychological distress. This scale is a common used in primary health care setting.

The researchers are used descriptive statistics to describe the results of data and determine the achievements of the study goals or not by using computer programs (SPSS v.17 and Microsoft Office Excel (2010)) to reveal the results.

\section{Results}

Table (1): Total number of health workers in the health facilities at Babylon Province

\begin{tabular}{|l|c|c|c|}
\hline & Total number of nurses only & Total number of other health workers & Total \\
\hline $\begin{array}{l}\text { All governmental health workers at } \\
\text { pandemic COVID-19 }\end{array}$ & 12450 & 12050 & 24500 \\
\hline Total & $\mathbf{1 2 4 5 0}$ & $\mathbf{1 2 0 5 0}$ & $\mathbf{2 4 5 0 0}$ \\
\hline
\end{tabular}

Table 2. Demographic characteristics of governmental health workers in health facilities at Babylon Province

\begin{tabular}{|c|c|c|c|}
\hline & & \multicolumn{2}{|c|}{$N=\mathbf{2 2 0}$} \\
\hline & & Frequency & Percent \\
\hline \multirow{2}{*}{ Gender } & Male & 163 & 74.1 \\
\hline & Female & 57 & 25.9 \\
\hline \multirow{3}{*}{ Age groups } & $\leq 30 \mathrm{yrs}$ & 151 & 68.6 \\
\hline & $30-45$ & 56 & 25.5 \\
\hline & More than 45 yrs & 13 & 5.9 \\
\hline \multirow{4}{*}{ Level education } & Post Graduate & 21 & 9.5 \\
\hline & College Graduate & 97 & 44.1 \\
\hline & Institute Graduate & 85 & 38.6 \\
\hline & Secondary School & 17 & 7.7 \\
\hline \multirow{4}{*}{ Specialty of health workers } & Medicine & 35 & 15.9 \\
\hline & Nursing & 106 & 48.2 \\
\hline & Other health workers & 63 & 28.6 \\
\hline & Support health workers & 16 & 7.3 \\
\hline \multicolumn{2}{|l|}{ Total } & 220 & 100 \\
\hline
\end{tabular}


Table 3. Social and duration of experiences of health workers at era of COVID-19 pandemic in health facilities at Babylon Province.

\begin{tabular}{|c|c|c|c|}
\hline & & \multicolumn{2}{|c|}{$N=220$} \\
\hline & & Frequency & Percent \\
\hline \multirow{4}{*}{ Marital status } & Married & 144 & 65.5 \\
\hline & Separated & 2 & 0.9 \\
\hline & Single & 73 & 33.2 \\
\hline & Widow & 1 & 0.5 \\
\hline \multirow{4}{*}{ Number of children } & Not had children & 112 & 50.9 \\
\hline & $1-2$ child & 62 & 28.2 \\
\hline & 3-4 child & 33 & 155 \\
\hline & More than 5 & 13 & 5.9 \\
\hline \multirow{5}{*}{ Place job } & Health sector & 17 & 7.7 \\
\hline & Hospital & 176 & 80 \\
\hline & Center of health office & 14 & 6.4 \\
\hline & Special centers & 8 & 3.6 \\
\hline & Voluntary work & 5 & 2.3 \\
\hline \multirow{6}{*}{$\begin{array}{l}\text { Previous Skills with endemic } \\
\text { Diseases }\end{array}$} & Not worked previously & 185 & 84.1 \\
\hline & $<1$ month & 6 & 2.7 \\
\hline & $1-6$ months & 14 & 6.4 \\
\hline & 6-12 months & 2 & 9 \\
\hline & $1-5 \mathrm{yrs}$ & 9 & 4.1 \\
\hline & $>5 \mathrm{yrs}$ & 4 & 1.8 \\
\hline \multirow{4}{*}{$\begin{array}{l}\text { Worked in Isolated units } \\
\text { previously }\end{array}$} & Not worked previously & 193 & 87.7 \\
\hline & $<1$ month & 6 & 2.7 \\
\hline & 1-6 months & 20 & 9.1 \\
\hline & $>5 \mathrm{yrs}$ & 1 & 0.5 \\
\hline \multirow{3}{*}{ Worked in Isolated units Now } & Not worked previously & 194 & 88.2 \\
\hline & $<1$ month & 7 & 3.2 \\
\hline & $1-6$ months & 19 & 8.6 \\
\hline \multicolumn{2}{|l|}{ Total } & 38 & 100.0 \\
\hline
\end{tabular}

Table 4. Level of General Mental Health of governmental health workers in health facilities at Babylon Province

\begin{tabular}{|c|c|c|c|c|c|c|}
\hline \multirow{2}{*}{ No. } & \multirow{2}{*}{ Items } & \multirow{2}{*}{ Ratings } & \multicolumn{2}{|c|}{$(\mathrm{N}=\mathbf{2 2 0})$} & \multirow{2}{*}{ M. } & \multirow{2}{*}{ S.D. } \\
\hline & & & F. & $\%$ & & \\
\hline \multirow{4}{*}{1.} & \multirow{4}{*}{$\begin{array}{l}\text { Been able to concentrate on what } \\
\text { you're doing? }\end{array}$} & - Better than usual & 42 & 19.1 & \multirow{4}{*}{2.97} & \multirow{4}{*}{0.68} \\
\hline & & - Same as usual & 134 & 6.9 & & \\
\hline & & - Less than usual & 39 & 17.7 & & \\
\hline & & - Much less than usual & 5 & 2.3 & & \\
\hline
\end{tabular}




\begin{tabular}{|c|c|c|c|c|c|c|}
\hline \multirow{2}{*}{ No. } & \multirow{2}{*}{ Items } & \multirow{2}{*}{ Ratings } & \multicolumn{2}{|c|}{$(\mathrm{N}=\mathbf{2 2 0})$} & \multirow{2}{*}{ M. } & \multirow{2}{*}{ S.D. } \\
\hline & & & F. & $\%$ & & \\
\hline \multirow{4}{*}{2.} & \multirow{4}{*}{ Lost much sleep over worry? } & - Not at all & 0 & 0 & \multirow{4}{*}{2.47} & \multirow{4}{*}{1.24} \\
\hline & & - No more than usual & 86 & 39.1 & & \\
\hline & & - Rather more than usual & 79 & 35.9 & & \\
\hline & & - Much more than usual & 55 & 25 & & \\
\hline \multirow{4}{*}{3.} & \multirow{4}{*}{$\begin{array}{l}\text { Felt you were playing a useful part } \\
\text { in things? }\end{array}$} & - More than usual & 40 & 18.2 & \multirow{4}{*}{3.03} & \multirow{4}{*}{0.61} \\
\hline & & - Same as usual & 150 & 68.2 & & \\
\hline & & - Less than usual & 26 & 11.8 & & \\
\hline & & - Much less than usual & 4 & 1.8 & & \\
\hline \multirow{4}{*}{4.} & \multirow{4}{*}{$\begin{array}{l}\text { Felt capable of making decisions } \\
\text { about things? }\end{array}$} & - More than usual & 50 & 22.7 & \multirow{4}{*}{3} & \multirow{4}{*}{0.73} \\
\hline & & - Same as usual & 128 & 58.2 & & \\
\hline & & - Less than usual & 34 & 15.5 & & \\
\hline & & - Much less than usual & 8 & 3.6 & & \\
\hline \multirow{4}{*}{5.} & \multirow{4}{*}{ Felt constantly under strain? } & - Not at all & 0 & 0 & \multirow{4}{*}{2.91} & \multirow{4}{*}{1.22} \\
\hline & & - No more than usual & 58 & 26.4 & & \\
\hline & & - Rather more than usual & 65 & 29.5 & & \\
\hline & & - Much more than usual & 97 & 44.1 & & \\
\hline & \multirow{4}{*}{$\begin{array}{l}\text { Been able to concentrate on what } \\
\text { you're doing? }\end{array}$} & - Better than usual & 1 & 0.5 & \multirow{4}{*}{2.5} & \multirow{4}{*}{0.52} \\
\hline & & - Same as usual & 110 & 50 & & \\
\hline & & - Less than usual & 108 & 49 & & \\
\hline & & - Much less than usual & 1 & 0.5 & & \\
\hline & & - Not at all & 11 & 5 & & \\
\hline & 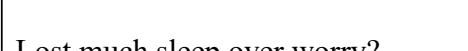 & - No more than usual & 37 & 16.8 & 3 & 08 \\
\hline & 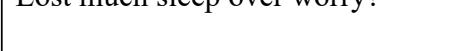 & - Rather more than usual & 113 & 51.4 & J & 0.0 \\
\hline & & - Much more than usual & 59 & 26.8 & & \\
\hline & & - More than usual & 58 & 26.4 & & \\
\hline & Felt you were playing a useful part & - Same as usual & 67 & 30.5 & 24 & 120 \\
\hline & in things? & - Less than usual & 0 & 0 & 2.4 & 1.20 \\
\hline & & - Much less than usual & 95 & 43.2 & & \\
\hline & & - More than usual & 19 & 8.6 & & \\
\hline & Felt capable of making decisions & - Same as usual & 45 & 20.5 & 167 & 1.07 \\
\hline & about things? & - Less than usual & 0 & 0 & 1.07 & 1.01 \\
\hline & & - Much less than usual & 156 & 70.9 & & \\
\hline & & - Not at all & 176 & 80 & & \\
\hline & & - No more than usual & 0 & 0 & 140 & 101 \\
\hline & Felt constantly under strain? & - Rather more than usual & 24 & 10.9 & 1.49 & 1.01 \\
\hline & & - Much more than usual & 20 & 9.1 & & \\
\hline & & - Not at all & 65 & 29.5 & & \\
\hline & Felt you couldn't overcome your & - No more than usual & 118 & 53.6 & 258 & 109 \\
\hline & difficulties? & - Rather more than usual & 0 & 0 & 2.50 & 1.09 \\
\hline & & - Much more than usual & 37 & 16.8 & & \\
\hline
\end{tabular}


Table 5. Overall Psychological Well-being among governmental health workers in health facilities at Babylon Province

\begin{tabular}{|l|c|c|}
\hline \multirow{2}{*}{ Psychological well-being G.H.Q } & F. & 220) \\
\cline { 2 - 3 } & 111 & 50.5 \\
\hline No Psychological Distress & 109 & 49.5 \\
\hline Psychological Distress & $\mathbf{2 2 0}$ & $\mathbf{1 0 0 . 0}$ \\
\hline Total & $\mathbf{2 0}$ \\
\hline
\end{tabular}

The table revealed (50.5\%) was have psychological distress (49.5\%) were have psychological distress.

Table (6): The relationship between Overall Psychological Well-being (GHQ) and Demographic Characteristics of governmental health workers in health facilities at Babylon Province

\begin{tabular}{|c|c|c|c|c|c|c|}
\hline \multirow{2}{*}{$(\mathrm{N}=\mathbf{2 2 0})$} & \multirow{2}{*}{ Demographic characteristics } & \multicolumn{2}{|c|}{ Overall Psychological Well-being } & \multicolumn{3}{|c|}{ Chi-Square tests } \\
\hline & & Less than usual & Same as usual & Value & df & Sig. \\
\hline \multirow{2}{*}{ Gender } & Male & 87 & 76 & \multirow{2}{*}{3.689} & \multirow{2}{*}{1} & \multirow{2}{*}{.05} \\
\hline & female & 22 & 35 & & & \\
\hline \multirow{3}{*}{ Age groups } & $<=30 \mathrm{yrs}$ & 75 & 76 & \multirow{3}{*}{2.197} & \multirow{3}{*}{2} & \multirow{3}{*}{.33} \\
\hline & $30-45$ yrs & 30 & 26 & & & \\
\hline & $>45$ yrs & 4 & 9 & & & \\
\hline \multirow{4}{*}{ Marital status } & Married & 71 & 73 & \multirow{4}{*}{3.133} & \multirow{4}{*}{3} & \multirow{4}{*}{.37} \\
\hline & Separated & 0 & 2 & & & \\
\hline & Single & 38 & 35 & & & \\
\hline & Widow & 0 & 1 & & & \\
\hline \multirow{4}{*}{ Education level } & College & 44 & 53 & \multirow{4}{*}{4.055} & \multirow{4}{*}{3} & \multirow{4}{*}{.26} \\
\hline & Institute & 46 & 39 & & & \\
\hline & Post Graduate & 13 & 8 & & & \\
\hline & Secondary School & 6 & 11 & & & \\
\hline \multirow{5}{*}{ Place Job } & Health Sector & 10 & 7 & \multirow{5}{*}{1.202} & \multirow{5}{*}{4} & \multirow{5}{*}{.88} \\
\hline & Hospital & 85 & 91 & & & \\
\hline & Office of Health & 8 & 6 & & & \\
\hline & Specialty center & 4 & 4 & & & \\
\hline & Voluntary Work & 2 & 3 & & & \\
\hline \multirow{4}{*}{ Specialty } & Medicine & 21 & 14 & \multirow{4}{*}{1.864} & \multirow{4}{*}{3} & \multirow{4}{*}{.61} \\
\hline & Nursing & 50 & 56 & & & \\
\hline & Other Health Workers & 30 & 33 & & & \\
\hline & Support Health Workers & 8 & 8 & & & \\
\hline \multirow{6}{*}{$\begin{array}{l}\text { Priovious Skills with } \\
\text { endemic Disease by } \\
\text { month }\end{array}$} & Not has worked previosuely & 94 & 91 & \multirow{6}{*}{2.840} & \multirow{6}{*}{5} & \\
\hline & $<1$ month & 4 & 2 & & & \\
\hline & $1-6$ months & 5 & 9 & & & 72 \\
\hline & 6-12 months & 1 & 1 & & & .12 \\
\hline & $1-5 \mathrm{yrs}$ & 3 & 6 & & & \\
\hline & $>5 \mathrm{yrs}$ & 2 & 2 & & & \\
\hline
\end{tabular}




\begin{tabular}{|l|l|c|c|c|c|c|}
\hline \multirow{2}{*}{$(\mathbf{N}=\mathbf{2 2 0})$} & \multirow{2}{*}{ Demographic characteristics } & \multicolumn{2}{|c|}{ Overall Psychological Well-being } & \multicolumn{3}{|c|}{ Chi-Square tests } \\
\cline { 3 - 6 } & & Less than usual & Same as usual & Value & df & Sig. \\
\hline \multirow{4}{*}{$\begin{array}{l}\text { Worked in Isolated units } \\
\text { previousely }\end{array}$} & Not has worked previosuely & 97 & 96 & & & \\
\cline { 2 - 4 } & $<1$ month & 3 & 3 & \multirow{3}{*}{1.787} & 3 & .61 \\
\cline { 2 - 4 } & $1-6$ months & 8 & 12 & & & \\
\cline { 2 - 4 } & $>5$ yrs & 1 & 0 & & & \\
\hline
\end{tabular}

\section{Discussion}

Most of health workers are participated in study are males (74\%) and their aged less than 30 years old (68\%) and had bachelor degree (44\%) (table 2). About $(65 \%)$ of participants are married and not had child $(50.1 \%)$ and they are not worked previously in isolated places or practical skills with epidemic diseases (84\%) (table (3)). In table (4) revealed the Level of general mental health of participants in the study according the items of general health questionnaire (GHQ-12 items) who work in health facilities at Babylon Province, but generally, half health workers are participated in study had highly incidence levels of psychological problems during the COVID-19 virus (table 5). Therefore, these result considered as a risk indicator if it is not treated and can be increased among them. These result consent with result of Zhang study $(2020)^{[1]}$. These the problems had a significant complication and impairment in social and occupational functioning; and feeling overwhelmed by the demands of everyday life on them ${ }^{[5]}$. The reasons for the psychological problems may be attributed to the many factors such as the initially inadequate awareness about COVID-19 and knowledge about how prevent it and control, as well as long-term of workload, and lack the medical protective equipment. In table (6) the researchers find significant relationship between the psychological problems and gender of health workers ${ }^{[8]}$ may due to the nature of male from female by how adopting and coping with the stress and events.

The researchers concluded to increase in psychiatric problems among health workers and may be elevated if it is not treated correctly. Decrease stigma, encouragement and training about how to reduce and faces stresses are very important. Finally, the support and available the medical equipment is important items to decrease the psychological problems on them.

Financial Disclosure: There is no financial disclosure.

Conflict of Interest: None to declare.
Ethical Clearance: After the administrative arrangements are completed, acceptance of health workers participants was sought for after explaining to them the aim of the study and inform them that all the information taken will be treated confidentially and it is for research purposes only,then taken the consent from them to participate in this study. Also, an ethical approval was obtained from ethical committee of research in Faculty of Nursing University of Kufa regarding confidentiality and anonymity of participants.

\section{References}

1. Zhang W, Wang k, Yin L, Zhao W, Xue Q., Peng M, Min B, Tian Q, Leng H, Du J, Chang H, Yang Y, Li W, Shangguan F, Yan T, Dong H. Mental Health and Psychosocial Problems of Medical Health Workers during the COVID-19 Epidemic in China, PsychotherPsychosom, Published online: April 9, 2020; 2-12.

2. Mishriky R S, and JishiA S. Mental Health Tool Kit, Johns Hopkins Aramco Health Care, 2020; 1(2).

3. Li Q, Guan X, Wu P, Wang X, Zhou L, Tong Y, et al. Early transmission dynamics in Wuhan, China, of novel coronavirus-infected pneumonia, N Engl J Med, 2020mar; 382(13): 1199-1207.

4. Day M. Covid-19: surge in cases in Italy and South Korea makes pandemic look more likely. BMJ, 2020, Feb; 368: 751.

5. World Health Organization [Internet]. Geneva: WHO statement on cases of COVID-19 surpassing 100 000, 2020, March 7; https://www.who.int/ newsroom/detail/07-03-2020-who-statementoncase-of-covid-19-surpassing-100-00.

6. Maryland Department of Health: Coronavirus Disease 2019 (COVID-19): Frequently Asked Questions About Mental Health, Updated June 5, 2020 .

7. Jansson M, Rello j.Mental Health in Healthcare Workers and the Covid-19 Pandemic Era: Novel 
Challenge for Critical Care, Journal of Intensive and Critical Care, 2020; 6(2:6), DOI: 10.36648/24718505.6.2.6

8. Guidelines COVID-19 Management: mental health and psychosocial support in covid-19, 2020 Updated On (24 March 2020); No.5.

9. Moghbelli H, Ellithy K, Eslami Z. SCCM COVID-19 Rapid-Cycle Survey 2 Report, on website, 2020 Mar.

10. Pappa S, Ntella V, Giannakas T, Giannakoulis VG, Papoutsi E, et al. Prevalence of depression, anxiety, and insomnia among healthcare workers during the COVID-19 pandemic: A systematic review and meta-analysis. Brain BehavImmun, 2020.

11. Pfefferbaum B, North CS. Mental Health and the Covid-19 Pandemic. N Engl J Med, 2020.

12. National Guideline and Standard Operating Procedure on Mental Health and Psychosocial Support in Disaster, NGSPMHPS, 2019; Edition $2^{\text {nd. }}$.

13. Al-Hamoody Astabrak A. Psychological problems among special educators in governmental institutes of handicap children, 2019; in pressed. 